\title{
Facilitating the 3D Indoor Search and Rescue Problem: An Overview of the Problem and an Ant Colony Solution Approach
}

\author{
H. Tashakkori *, A. Rajabifard, M. Kalantari
}

Centre of Disaster Management and Public Safety, Department of Infrastructure Engineering, The University of Melbourne, Parkville, Vic 3010, Australia - stashakkori@ student.unimelb.edu.au, (abbas.r, mohsen.kalantari)@unimelb.edu.au

KEY WORDS: Indoor Search and Rescue, 3D Indoor GIS, Emergency Response, Indoor Path Finding, Ant Colony Optimization

\begin{abstract}
:
Search and rescue procedures for indoor environments are quite complicated due to the fact that much of the indoor information is unavailable to rescuers before physical entrance to the incident scene. Thus, decision making regarding the number of crew required and the way they should be dispatched in the building considering the various access points and complexities in the buildings in order to cover the search area in minimum time is dependent on prior knowledge and experience of the emergency commanders. Hence, this paper introduces the Search and Rescue Problem (SRP) which aims at finding best search and rescue routes that minimize the overall search time in the buildings. 3D BIM-oriented indoor GIS is integrated in the indoor route graph to find accurate routes based on the building geometric and semantic information. An Ant Colony Based Algorithm is presented that finds the number of first responders required and their individual routes to search all rooms and points of interest inside the building to minimize the overall time spent by all rescuers inside the disaster area. The evaluation of the proposed model for a case study building shows a significant improve in search and rescue time which will lead to a higher chance of saving lives and less exposure of emergency crew to danger.
\end{abstract}

\section{INTRODUCTION}

The time period right after a disaster strikes is the most critical time for saving victims' lives, battling the consequences of the chaotic situation, and minimizing the damage caused to both public and properties (Diehl and van der Heide, 2005). Studies have shown only a small delay of 5-10 minutes of arrival of rescue services can hugely impact the fire damage and risk to people and first responders' lives in structural fires (Mattsson and Juås, 1997). In fact, firefighter injuries are a main concern of emergency bodies (NFPA, 2013) and although there has been great focus in research on public evacuation in buildings (Tang et al., 2014), importance of models aimed to navigate first responders through built environments and providing them with emergency critical information has been underestimated.

In the current practice, firefighters know little about the fire and the building until they get to the scene where they have to quickly evaluate the situation and take actions accordingly. In fact, lack of timely information reduces their preparedness ability to act rapidly when confronted with an incident. However, first responders should not have to physically enter a burning building to gain critical information regarding that building (Evans, 2003) and such information should be readily available to them in their trucks while approaching the scene, giving them detailed situational awareness of the incident area (Tashakkori et al., 2015b).

Searching the incident building for possible victims and location of fire is one of the major tasks undertaken by first responders after an incident occurs. In fact chances of finding live victims in residential buildings under dangerous situations depends on the speed of the primary search done in the building (Bricault, 2006). Considering the importance of this thorough search in the building, an important research problem is how the first responder crew should be dispatched inside the building in order to minimize the search and rescue time. This could be quite a complicated task due to various entrances, exits, staircases, and elevators in the building which creates various choices and difficulties in choosing the paths for rescuers and as of now, decisions on dispatching the crew depends on previous knowledge and experience of the incident managers.

3D indoor models have proven to enhance the localization, orientation, and visual cognition of the indoor environments (Rakkolainen and Vainio, 2001) and also improve the speed of rescue for indoor operations (Kwan and Lee, 2005; Tashakkori et al., 2015a). Thus, research has attempted to integrate current geographical information systems with geometric and semantic information of building utilities from 3D Building Information Models (BIM) (Eastman et al., 2011) for indoor way finding (El-Mekawy et al., 2012; Isikdag et al., 2013; Thill et al., 2011). However, only a few research have considered indoor information that is critical for emergency response operations and can be used for indoor emergency applications (Chen et al., 2014; Tashakkori et al., 2015a). Furthermore, accurate indoor route finding for emergency crew depends on availability of detailed indoor information aimed at this problem and simply routing them through shortest paths is not sufficient.

To address these issues, the Search and Rescue Problem (SRP) is introduced in this paper which uses an integrated indoor GIS Geometric Network Model (GNM) to facilitate the decision making of how an emergency crew should be dispatched in case of an indoor incident to search the building efficiently. The Search and Rescue Problem is defined as finding the optimum routes for the minimum number of first responders to search and visit all points inside a building for possible victims in the quickest time possible. Considering the various access points of a building, accessibility of spaces, and traversing speed in different segments of the building, the solution to SRP would suggest best routes including which door to enter and which 
door to exit for each responder to optimize the overall performance of all the responders in the scene.

A 3D indoor GIS representation of building structures aimed at emergency response is integrated with the indoor Geometric network Model (GNM) to create the network dataset for the Search and Rescue Problem. SRP is formulated as an integer linear programming problem making it an np-hard problem where the solution can't be found in polynomial time. Therefore, an ant colony based approach is proposed to solve it and find near optimum solutions for the problem. Formulating and solving this problem will help decision makers to have a close estimate of the minimum number of crew needed to be taken to the incident scene, and by having detailed routes for each and every person, they can maximize efficiency of first response operations by minimizing the time each first responder spends indoors. The proposed model is evaluated using a case study building and the results show significant improvement of search time.

In the rest of the paper, Section 2 will discuss search and rescue operations in complex indoor environments and review related work in the field. Then Section 3 will describe the creation of the integrated indoor/outdoor route network for the Search and Rescue Problem. Formulation of SRP and the ant colony based approach will be presented in Section 4. Lastly, the efficiency of the proposed model will be evaluated on a case study in Section 5. And, finally Section 6 will conclude the paper and discuss future research and improvements.

\section{SEARCH AND RESCUE IN COMPLEX INDOOR ENVIRONMENTS}

Complexity of buildings, high rise structures and underground facilities makes emergency management in such areas quite challenging (Safety Health and Survival Section International Association of Fire Chiefs, 2012). . In the current practice, a 360 degree size-up of the incident and an assessment of the structure and the blueprints of the buildings and physical spaces is conducted rapidly after arrival of the emergency crew and an action plan is developed accordingly on the site. Although, some building owners provide the fire brigades with their $2 \mathrm{D}$ CAD maps, such maps are difficult to interpret quickly, lack detailed emergency information and are not available for all the structures and residential areas. For the primary search operations, firefighters are trained to use the right or left hand technique to keep them oriented. They unreel ropes called lifelines and fire hoses to find routes along the way and occasionally use thermal cameras in poor visual conditions (Safety Health and Survival Section International Association of Fire Chiefs, 2012). Still, getting lost in the building, disorientation, miscommunication of locations and spaces, and not knowing the paths cause huge amounts of casualties in indoor structures (Safety Health and Survival Section International Association of Fire Chiefs, 2012). In such events, unavailability of detailed geometric and semantic maps plays a major role.

Various research have focused on new approaches to facilitate indoor emergency operations. Indoor navigation systems are becoming more advanced in locating and tracking people in indoor environments, however they still have limitations when used by emergency responders due to the challenging situations they confront during emergencies (Fischer and Gellersen, 2010). Virtual reality training of fire fighters has shown to aid rescuers in performing the rescue tasks quicker (Bliss et al., 1997) and most recently serious game platforms with BIM representations and fire simulations have been proposed for training experts on hazards they may confront on the fire scene (Diez et al., 2016).

Still, searching the interiors of a building could be a complicated task considering the complexity of the structure, unfamiliarity with the areas, and visual distractions. In fact, Indoor travel times are proved to be higher compared to outdoors due to slower walking speed, uncertainty of paths, navigation restrictions caused by bounded walls and limitation in navigable areas (Kwan and Lee, 2005).

To overcome the path finding issues in indoor structures, a great body of research has focused on routing and navigating inside buildings in the recent years (Afyouni et al., 2013; Fallah and Apostolopoulos, 2013). Most of the literature focuses on how to traverse between two points especially for evacuation and optimal route finding to move people from an affected area to safer locations (Tang et al., 2014). Evacuation strategies proposed for indoors are mainly based on travelled distance in the structure (Fallah and Apostolopoulos, 2013), leaving out other main structural and semantic parameters. Isikdag et al. (2013) and Lin et al. (2013) introduce the use of BIM for integrating semantic information of building components along with the geometric information to map the building into a planar grid and shortest path algorithms are used for path finding. Real time information like congestion detectors can be used to increase routing awareness inside structures (Chen et al., 2011; Inoue et al., 2008). Projects like FireGrid are also focusing on next-generation emergency response systems by gathering realtime data from intelligent buildings (Berry et al., 2005; Han et al., 2010). All the approaches mentioned above mainly target public evacuation in emergency situations but don't reflect first responders' requirements during indoor incidents.

Search and Rescue as a widely used term among fire services defines the act of firefighters looking for something which could be the location of fire, potential victims, or interior fire conditions. And Rescue is defined as the act of firefighters removing a victim physically from the dangerous area to a safe location (Bricault, 2006). In fire academies, fire fighters are skilled on how to perform primary search using the right hand or left hand search techniques. The main objective is for the firefighter to search the area thoroughly while keeping themselves oriented with the environment. The sooner and quicker the primary search is executed, the chances of rescuing more live people increases. Therefore, this paper introduces and solves SRP to take out the guessing and risk factor of path decisions in indoor environments. Wu \& Chen (2012) address indoor search and rescue problem in a relaxed form in which a spatio-temporal analysis method is proposed to find a single fire-fighting route for searching the points. Furthermore, in their approach a Traveling Salesman Problem (TSP) is formulated for the points that need to be searched inside the building and the minimum response time is found to search all the assigned points within a building by one rescuer. However, the GNM used in their work is not integrated with the outdoor environment and doesn't consider existence of multiple entering and exiting points as well as multiple responders in the problem. Thus formulating the problem as a TSP, their solution is limited in how it can be used for 3D structures.

To solve SRP, this paper presents an ant colony based algorithm. Ant colony optimization (ACO) is a strong category of metaheuristic algorithms based on the real ants behaviour to forage food (Maniezzo et al., 1991). ACO algorithms have promising results for TSP (Dorigo et al., 2010) and its 
generalized form Vehicle Routing Problems (VRP)(Gambardella et al., 1999) and more recently it is being used for optimizing evacuation routes inside buildings (Sabri et al., 2016). SRP's accuracy relies heavily on having a detailed underlying route network that would integrate the outdoor road network with the indoor graph. As 3D GIS representations of the internal structures are proved to improve speed of rescue operations (Kwan and Lee, 2005; Tashakkori et al., 2015a), SRP will be based on a detailed 3D Indoor GIS in this paper explained in the following section.

\section{3D INDOOR GIS INTEGRATED GEOMETRIC NETWORK MODEL}

To have an efficient SRP algorithm which can accurately find best paths for responders inside structures, indoor environments need to be modelled precisely to reflect all the navigable locations in the building. Horizontal slabs, vertical access points like stairs and elevators, entrance and exit points, rooms and corridors, hazard points, accessible and non-accessible areas, etc. should all be well represented in the navigable model. Distance based route graphs that only rely on the lengths of edges for finding shortest path algorithms are not sufficient for emergency response requirements in indoor areas. Moreover, 3D geometric information of the indoor elements such as door and window heights, corridor wideness, etc. as well as semantic information such as accessibility of specific points, type of doors (entrance or exit), type of windows (whether they can be used as exit points), hazard status in rooms, etc. play a crucial role in how the route graphs are defined (Lin et al., 2013). Hence, new approaches have been developed for indoors based on the $3 \mathrm{D}$ aspects of such structures which contrary to traditional 2D representations contain semantically rich 3D information essential for path finding and navigation (Chen et al., 2014; Isikdag et al., 2013; Tashakkori et al., 2015a).

As the time spent by first responders on the scene using the models is very short, the information presented to them regarding the building must be chosen carefully (Jones et al., 2005). To improve the applicability of the indoor models for navigation applications new methodologies for indoor modelling have been proposed that integrate geometric and semantic information required for indoor navigation from BIM and CityGML (El-Mekawy et al., 2012; Isikdag et al., 2013). To make such models more applicable to indoor emergency cases, 3D IESM (Indoor Emergency Spatial Model) proposes modifying IFC to contain mission critical data required by emergency responders in the scene and thus can be used to enrich route graphs for emergency response specific applications (Tashakkori et al., 2015a). IESM is based on the thorough investigation of first responder requirements on scene which is translated into a conceptual data model containing building structural components, emergency components, semantic information, dynamic inputs, and outdoor emergency information. As IESM contains the complete indoor information required by emergency responders, it will be used in this paper for creating the SRP route graph.

The BIM representation of the building (Figure 1) and the 2D floor plans can be used to structure the 3D indoor Geometric Network Model using Lee's node-relation structure. Rooms, doors, and windows that can be used as exit points form the nodes of the network; and corridors, staircases and elevators form the edges, connecting floors together (Figure 2). The geometric network model allows visualization and cost computation of the route graph which is explained below. Also, the detailed attributes and semantic information of the utilities in the building are integrated from IESM by labelling the nodes and edges in the GNM. All doors and windows that have the potential to be used as access points to the building are connected directly to the outdoor road network to enable the seamless connection. The network model was extracted manually from the IFC and IESM representation to reflect all the required attributes.

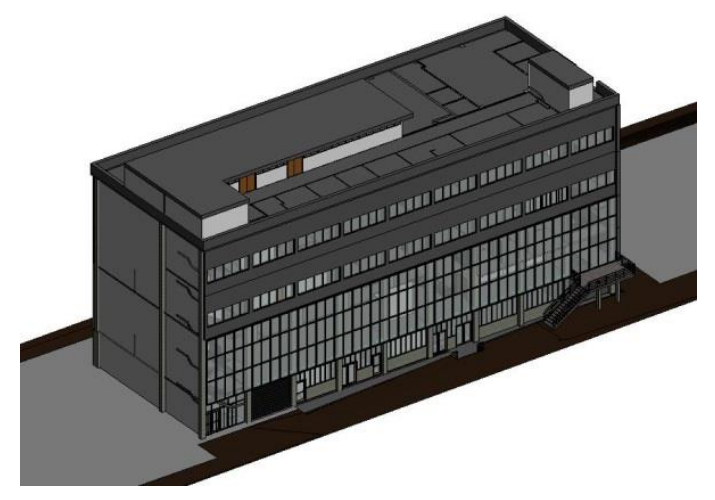

Figure 1. BIM representation of building

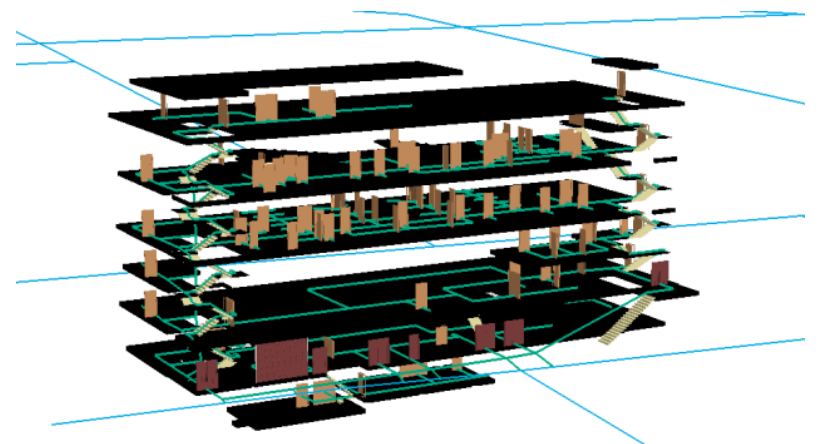

Figure 2. 3D Indoor GIS integrated GNM creation

Table 1 IESM integration into the indoor GNM

\begin{tabular}{|c|c|}
\hline GNM Feature & \begin{tabular}{|l} 
Labels \\
\end{tabular} \\
\hline Node & $\begin{array}{l}\text { Segment Type (door, window, roof, } \\
\text { slab, stair, elevator, etc.) } \\
\text { Floor no } \\
\text { Has Hazard } \\
\text { Accessibility } \\
\text { Ownership Information } \\
\text { Is fire door }\end{array}$ \\
\hline Edge & $\begin{array}{l}\text { Segment type (slab, stair, elevator) } \\
\text { Floor no } \\
\text { Has Hazard } \\
\text { Accessibility } \\
\text { Traversing Time } \\
\text { Traversing Distance } \\
\text { Fire Resistance }\end{array}$ \\
\hline
\end{tabular}

The labelling of the SRP GNM for integration of IESM is done according to the building features. For simplicity, only attributes effective on route optimization in SRP have been considered. Some attributes are common among all the features like floor number which allows categorizing the network based on the floor they belong to. Thus this attribute is attached as a label to the nodes and edges in the network. However, other attributes are more specific to a particular feature. For instance, 
nodes that represent doors in the network carry special attributes to identify if they are room doors or main exit and entrance doors to the building. They are also labelled as fire doors or fire resistant if that is the case. Also, as described in IESM, each space in a building is mapped to a node in the graph which holds critical information such as if it contains hazardous material, type of the hazard, owner information, as well as occupancy information. All of this information can be labelled on the node and stored in the indoor GNM for emergency response path finding in the network (Table 1). Therefore, if it is important for first responders to quickly locate the hazard points in the building and search those points first, they can easily identify them and run the SRP for those points first.

Traversing speed of various types of segments in the building differs with one another and the time traversed is not in direct relation with distance. While elevators have the quickest movement speed inside a building, walking speed on slabs or horizontal spaces is slower compared to elevators and quicker compared to vertical stair segments. To allow discovery of quickest rescue paths, traversing time of edges are found using eq. 1

$$
t_{i j}=\frac{d_{i j}}{s_{i j}}
$$

Where:

$$
\begin{aligned}
& t_{i j} \text { is the traversing time of edge }(i, j) \\
& d_{i j} \text { is the distance of edge }(i, j) \\
& s_{i j} \text { is the speed of edge }(i, j)
\end{aligned}
$$

Here, average travelling speeds on the road are assumed to be $40 \mathrm{~km} / \mathrm{h}$. Walking speed on horizontal segments is considered 25 meters per minute while the speed for climbing stairs is 15 meters per minute and elevators move at an average of 6 meters per second. Although, the use of elevators is discouraged in emergency situations some research show that they are a necessity and the usage is unavoidable (Aleksandrov et al., 2015).

Accordingly, the cost of each edge is a function of time and its accessibility derived from the equation below:

$$
c_{i j}=t_{i j} \times a_{i j}
$$

Where:

$$
a_{i j}= \begin{cases}1, & (i, j) \text { is accessible } \\ \infty, & (i, j) \text { is not accessible }\end{cases}
$$

Here $c_{i j}$ is the cost of each edge from node $i$ to $j, t_{i j}$ is the speed and $a_{i j}$ shows the accessibility of the edge. If the edge is not accessible in the graph meaning the location is blocked or inaccessible in the building, it is impossible to use that edge, so the cost of traversing it will be set to infinity. Thus, the total traversing time of a route would be calculated using the equation below:

$$
\begin{aligned}
\sum_{i, j \in v} x_{i j} \frac{d_{i j}}{s_{\text {horizontal }}} & +y_{i j} \frac{d_{i j}}{s_{\text {stair }}}+z_{i j} \frac{d_{i j}}{S_{\text {elevator }}} \\
& +r_{i j} \frac{d_{i j}}{s_{\text {road }}}
\end{aligned}
$$

Where:

$$
\begin{aligned}
x_{i j} & = \begin{cases}1, & \text { if }(i, j) \text { is used on tour and type }=\text { slab } \\
0, & \text { otherwise }\end{cases} \\
y_{i j} & = \begin{cases}1, & \text { if }(i, j) \text { is used on tour and type }=\text { stair } \\
0, & \text { otherwise }\end{cases} \\
z_{i j} & = \begin{cases}1, & \text { if }(i, j) \text { is used on tour and type }=\text { elevator } \\
0, & \text { otherwise }\end{cases} \\
r_{i j} & = \begin{cases}1, & \text { if }(i, j) \text { is used on tour and type }=\text { road } \\
0, & \text { otherwise }\end{cases}
\end{aligned}
$$

\section{SEARCH AND RESCUE PROBLEM}

\subsection{SRP Formulation}

The problem of indoor search and rescue can be defined as follows: Given a network dataset of a building with a graph $G=(V, A)$, where $V$ is the set of $N$ nodes (vertices) and $A$ is the set of arcs (edges) and $C=\left(c_{i j}\right)$ is the cost associated with traversing each edge, where $(i, j) \in A$, let there be $k \in$ $\{1,2, \ldots, K\}$ responders that are located on the street closest to the building. There are $\mathrm{M}$ points of entry to the building that can be either used for entrance or exit which we generally refer to as access nodes. The remaining nodes (rooms, corridors, etc.) that are to be visited are called intermediate nodes. The Search and Rescue Problem (SRP) consists of finding tours for K responders, who all start from an access node and finish at an access node together visiting all intermediate nodes such that the total time of visiting the nodes is minimized.

Let's consider the set of nodes in the network to be $V=$ $\{0,1,2, \ldots, M, \ldots, N\}$. The problem should be formulated to suggest best access nodes for entering and exiting the building in order to maximize routing efficiency. Therefore, we propose adding an extra virtual node that is connected to all access points. We refer to this node as a depot node where all responders start and finish their traversing path. All the edges connecting the depot node to the access nodes will have the same cost of zero which forces all responders to enter the building from an access node and finish their route by exiting through an access node. Node 0 is regarded as the depot node, and nodes $\{1,2, \ldots, M\}$ are the access nodes. The rest of the nodes $\{M+1, \ldots, N-1, N\}$ are the points that should be visited in the graph. As seen in Figure 3, all the doors in the building that could be used for entering or exiting the building are connected to a virtual node (the red node) which will set the start and finishing of all routes found in the building.

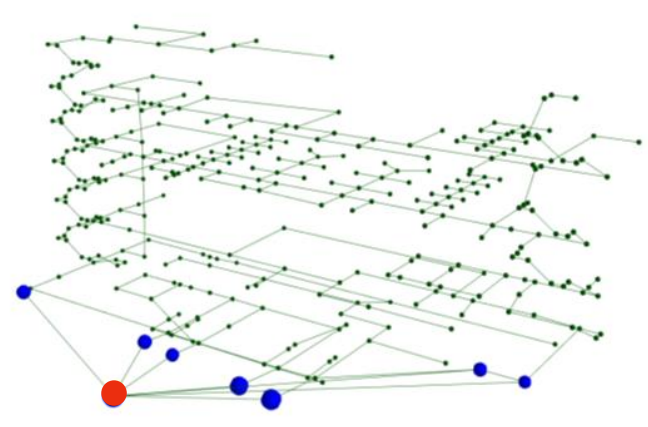

Figure 3. Search and Rescue Model Initiation

As such, solving SRP would be the same as finding the solution that minimizes the following objective function: 
$\operatorname{minimize} \sum_{k \in K} \sum_{\substack{i, j \in V, i \neq j}} c_{i j} x_{i j}^{k}$

Such that:

$$
\sum_{\substack{i, j \in V, i \neq j}} c_{i j} x_{i j}^{k}<t_{\max }
$$

Where:

$$
x_{i j}^{k}=\left\{\begin{array}{cc}
1 & \text { if } \operatorname{arc}(\mathrm{i}, \mathrm{j}) \text { is used by responder } \mathrm{k} \\
0 & \text { otherwise }
\end{array}\right.
$$

The objective function minimizes the total travelling time of all first responders in completing their designated routes to search the building. Each route is assigned to one responder $k$ and the cost of the route corresponds to the sum of traversing time of all the arcs forming the route. Constraint (5) limits the maximum time $\left(\boldsymbol{t}_{\max }\right)$ a rescuer can spend indoors without risk to his/her life and controls the balance of routes.

\subsection{Ant Colony Based Algorithm for SRP}

To solve SRP an Ant Colony Optimization (ACO) approach is used. ACO is a metaheuristic based on the behaviour of real ants which holds knowledge of past actions and distance to other locations in the network to find solutions to combinatorial optimization problems (Marco Dorogi, Mauro Birattari, 2006). The algorithm is based on the communication among ants using a chemical called pheromone. During the running iterations of the algorithm, shorter paths will have a higher concentration of pheromone and the colony will converge towards those paths making longer paths less reinforced. The evaporation of pheromone in time makes less desirable routes more difficult to detect allowing exploration of the entire search area and escaping from local optimums.

The ACO heuristic developed for SRP considers each ant in the colony to represent a complete tour of all rescuer paths inside a building and is constructed incrementally selecting rooms and points of interest in the building until all these points have been visited. In each iteration of the algorithm each ant searches the network to find the best routes for minimum number of rescuers to minimize the total traversal time considering their capacity.

Initially each ant starts from the depot and the set of points visited in its tour is empty. The ant selects the next point to visit in the graph based on the probability retrieved from eq.6. In this equation $\tau_{i j}$ is the amount of pheromone on the arc, $\eta_{i j}$ is the inverse of distance, and $\alpha$ and $\beta$ reinforce each of these parameters. The probability $p_{i j}$ is calculated for each of the points that have not yet been visited by the ant stored in memory $M_{k}$. In this case the ant selects the arc with the highest value from eq. 6 favouring shorter paths with higher levels of pheromone.

$$
p_{i j}=\frac{\left(\tau_{i j}\right)^{\alpha}\left(\eta_{i j}\right)^{\beta}}{\sum_{u \notin M_{k}}\left(\tau_{i j}\right)^{\alpha}\left(\eta_{i j}\right)^{\beta}}
$$

If the addition of the new point will violate the capacity constraint, the ant will return to the depot by using the closest exit door. And from there it will start a new path i.e. adding a new rescuer. The new path will then start from the depot moving towards the next unvisited point in the building. Once all ants have found a complete tour in the iteration, the pheromone levels are updated on the tours based on the quality of the tour; enforcing more pheromone on better tours.

\section{PERFORMANCE EVALUATION OF SRP}

\subsection{Implementation of SRP}

To evaluate SRP's performance, we implemented it for a test case geo-located building using Python 2.7.10. The IESM integrated Indoor GNM is extracted from the polyline shapefile of the building and the Mayavi package was used for the 3D scientific data visualization. The building has four floors, a rooftop and a basement with a graph consisting of 350 vertices and 377 undirected weighted edges, eight main entrances to the building, two staircases and an elevator. As the indoor GNM is integrated with IESM, various features and building utilities can be retrieved and visualized on the model as seen in Figure 4. The $3 \mathrm{D}$ visualization of the building and its main utilities would result in improved situational awareness of the indoor environments and assist first responders with the planning of the emergency procedures.

The ant colony algorithm is implemented as described above and is run for various scenarios to reflect its efficiency. The following section will discuss the results achieved from the implementation of the algorithm.

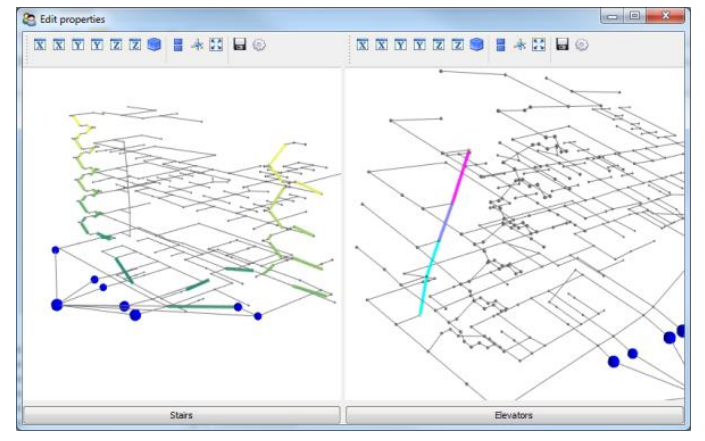

Figure 4. Visualization of the integrated indoor GNM

\subsection{Case Study Results}

Disaster environments are highly complex areas that are subject to dramatic changes in short times and various variables and structural parameters can affect the decision making in the scene. However, the objective of our proposed approach is to facilitate route finding and increase movability for first responders in the short time right after an indoor incident occurs where currently much of the information is not available. Thus, providing decision makers with awareness of the routes and indoor areas while travelling to the scene in their trucks; minimizing the uncertainty routing time inside buildings.

By setting the maximum time a rescuer is allowed to spend indoors, running the algorithm for the case study building will return the number of first responders required and best paths for each of them to search the entire building in minimum time. The paths get visualized on the 3D building for each person as shown in Figure 5. The coloured points in the visualization represent the doors (access nodes) in the building while the red point connected to the rest of the doors is the depot node located on the closest road to the building, linking the indoor and outdoor areas. 
In this scenario, the maximum capacity time a rescuer can spend inside the building before exiting it is assumed 900 seconds or 15 minutes. This assumption is based on the oxygen tank of a Self-Contained Breathing Apparatus (SCBA) which usually can last for 30 minutes. However this time is based on an average mail breathing speed and under the heavy activities of a firefighter this can only last between 6 to 21 minutes (Marino, 2006).

As the results show, a minimum of 6 rescuers will be required to search the entire building following the given paths to minimize the overall search time. As shown in the 6 paths found, each start from an entrance point to the building, search the unvisited points and exit the building from an exit point. As the figures show, the algorithm tends to visit the nodes in a floor based clustered approach to avoid overlap as much as possible (overlap is unavoidable due to limited egress points).

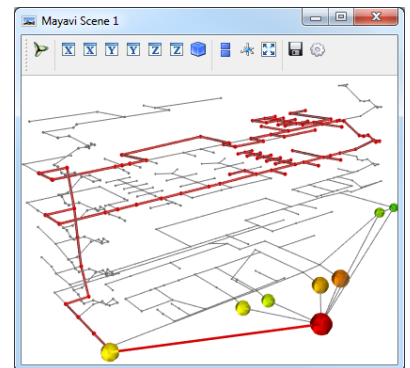

a) Search Route \#1

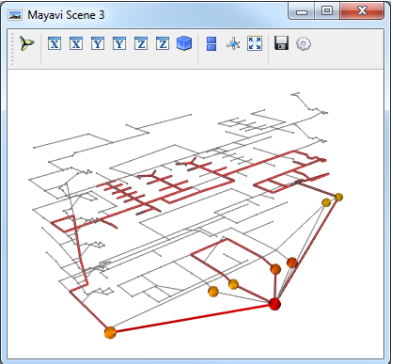

c) Search Route \#3

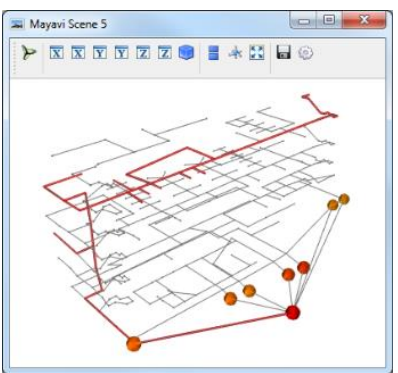

e) Search Route \#5

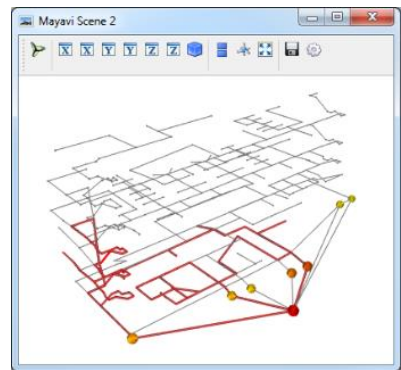

b) Search Route \#2

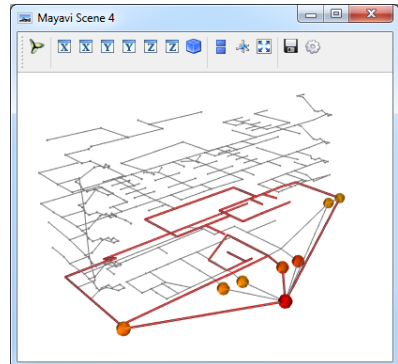

d) Search Route \#4

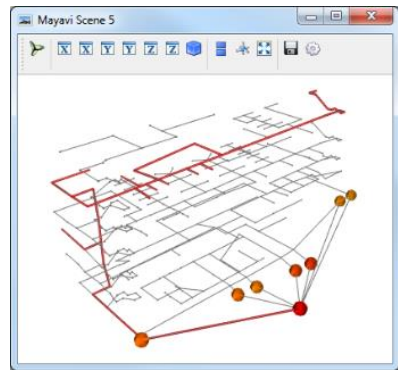

f) Search Route \#6
Figure 5. SRP Search Paths

The evolution of the algorithm during the iterations until it converges to the best solution for this scenario is shown in Figure 6 . As the chart illustrates, the aggregated time spent by all rescuers decreases from 8466 seconds to 5310 seconds as the algorithm improves. Similarly, the number of rescuers decreases from 9 to 6 people. Also as Figure 7 depicts, as the algorithm evolves, the routes found for rescuers become more balanced in terms of the time it takes to complete a route thus resulting in less dispersed routes.

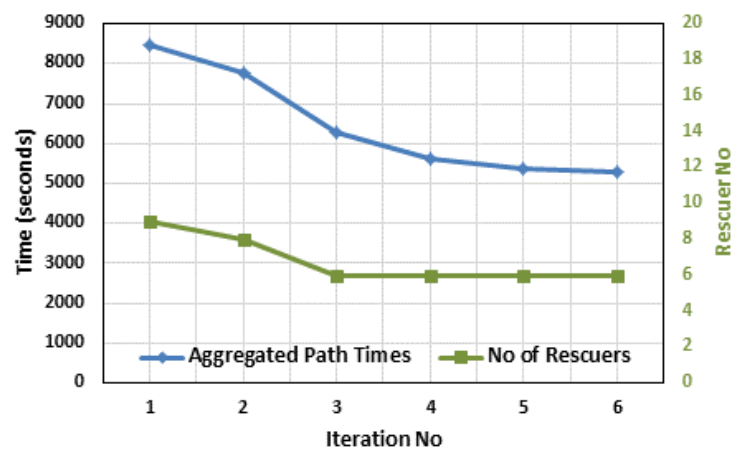

Figure 6. Finding minimum no. of rescuers and time during the iterations

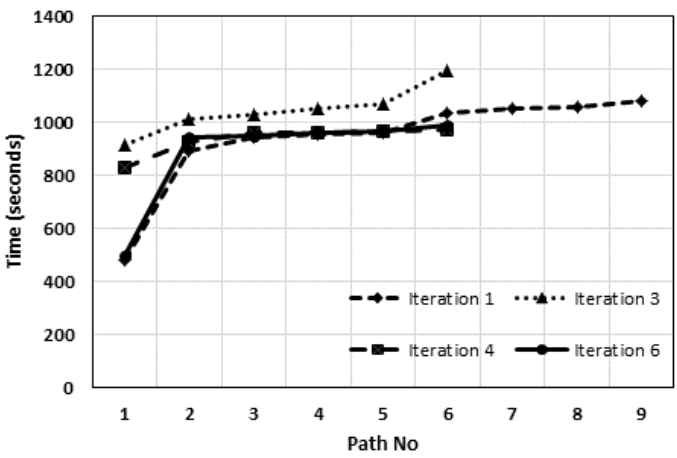

Figure 7. Solutions moving towards balanced routes

Running the algorithm under different maximum indoor time capacity constraints for first responders ranging between 5 minutes and 80 minutes, the results show that increasing the rescuers would lead to increased aggregated search time for rescuers in the building. This would mean the total travel time of all the rescuers would be increased. Similarly, increasing the number of rescuers for searching the indoor areas will lead to increased total traversal distances by all the rescuers. This is mainly because increasing the rescuers would mean more overlap in the routes since the number of egress points (stairs, elevators) are limited in the network. As illustrated in Figure 8 and Figure 9, although the increase in the time and distance are smooth for the first 6 rescuers, they increase hugely after a threshold of 8 rescuers is hit. However, the search time for the entire building is not improved much after this threshold mainly because of increased overlap of the routes (Figure 10). Figure 10 shows the maximum time for the Search and Rescue Problem in order to visit all the points inside the building. As the chart shows, the search time decreases by increasing the number of first responders for searching since search will be undertaken in a parallel way. If only one rescuer would do the entire search, this would take him/her 78 minutes. However, adding a second rescuer will decrease the search time to 41 minutes and adding a third person will decrease this to 34 minute and further rescuers will decrease the search time smoothly. Although having 8 rescuers would decrease the search time to 13 minutes, adding further rescuers wouldn't improve the speed anymore. As every minute counts in disaster management, improvement from 78 minutes to 13 minutes is a huge difference and can mean saving more lives and less exposure of rescuer lives to danger. 


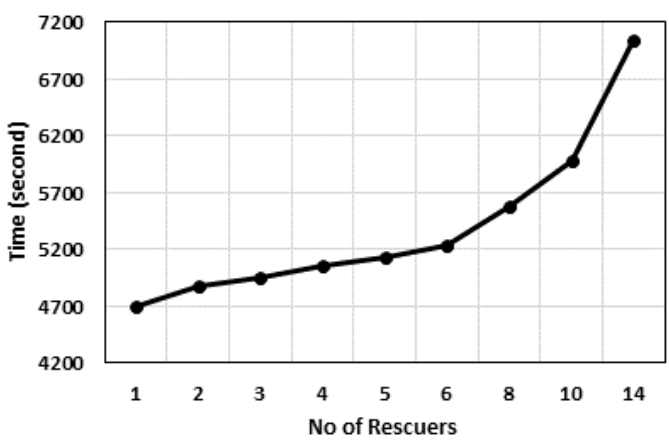

Figure 8. Aggregated search time

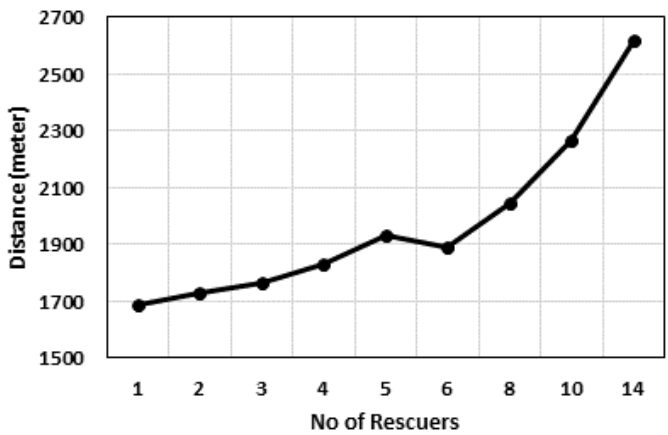

Figure 9. Aggregated distance travelled

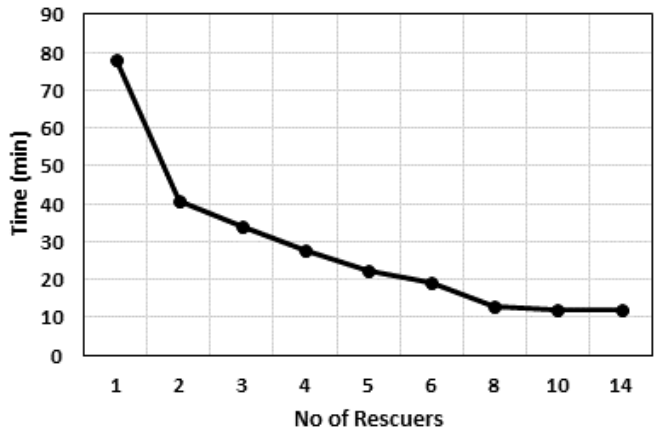

Figure 10. Time to finish Search

\section{CONCLUSION}

To assist incident managers in disseminating their crew for the search and rescue operations in an indoor incident, this paper introduced the Search and Rescue Problem (SRP) and an ant colony optimization based algorithm for solving it. To assure the accuracy and applicability of the algorithm for finding the routes, the indoor navigable graph is built upon a BIM integrated 3D indoor GNM which includes geometric and semantic information of the features inside the building that impact first responder navigation. Our proposed approach finds the minimum number of rescuers and individual routes for each one to search the building for possible victims and dangers in minimum time considering the capacity limitations of the crew. The results can serve a great input for incident managers in facilitating their decision making for indoor incidents by improving their situational awareness of the environment. To show the efficiency of the algorithm, it was implemented and tested for a case study and the results confirm our claim. The results clearly show the decisions of the number of rescuers and dispatching them can impact the response time hugely. While searching a four storey building progressively with one path to cover all the areas can take around 78 minutes, the search process can be broken into 8 individual paths using our proposed system to improve the search time to only 13 minutes. This is achieved by knowledge of the indoor network and building settings and minimizing the overlap of the routes.

Such improvement in route decision making can be effectively used by incident commanders and first responders as a 3D visualization platform available on handheld or head mounted devices that can be used even before arriving at the scene. Furthermore, the incident commanders will be able to better communicate path, routes and locations that should be searched by the crew members since he/she will have a clear view of the indoor areas without entering it physically. Also serious game platforms can use the model for training purposes.

Although providing this level of knowledge of the buildings can improve decision making vastly, it is important to remember that emergencies are very dynamic situations where areas are subject to blockage or collapse and are not always accessible through time. Considering the dynamics of the emergencies and the availability of real time information from intelligent buildings is a main aspect of how rescuer routes are identified which is our main focus for future research.

\section{REFERENCES}

Afyouni, I., Ray, C., Claramunt, C., 2013. Spatial models for context-aware indoor navigation systems: A survey. J. Spat. Inf. Sci. 4, 85-123. doi:10.5311/JOSIS.2012.4.73

Aleksandrov, M., Rajabifard, A., Kalantari, M., Tashakkori, H., 2015. Evacuation Time in Tall High-Rise Buildings, in: 2nd International Conference on Information and Communication Technologies for Disasater Management. Rennes, France.

Berry, D., Usmani, A., Torero, J.L., Tate, A., McLaughlin, S., Potter, S., Trew, A., Baxter, R., Bull, M., Atkinson, M., 2005. FireGrid: Integrated emergency response and fire safety engineering for the future built environment FireGrid technologies.

Bliss, J.P., Tidwell, P.D., Guest, M.A., 1997. The effectiveness of virtual reality for administering spatial navigation training to firefighters. Presence 6, 73-86.

Bricault, M., 2006. Residential Search and Rescue: Methodology. Fire Eng. 159.

Chen, L.C., Wu, C.H., Shen, T.S., Chou, C.C., 2014. The application of geometric network models and building information models in geospatial environments for fire-fighting simulations. Comput. Environ. Urban Syst. 45, 1-12. doi:10.1016/j.compenvurbsys.2014.01.003

Chen, Y., Sun, L., Wang, F., Zhou, X., 2011. CongestionAware Indoor Emergency Navigation Algorithm for Wireless Sensor Networks. 2011 IEEE Glob. Telecommun. Conf. GLOBECOM 2011 1-5. doi:10.1109/GLOCOM.2011.6134571

Diehl, S., van der Heide, J., 2005. Geo Information Breaks through Sector Think, in: van Oosterom, P., Zlatanova, S., Fendel, E. (Eds.), Geo-Information for Disaster Management SE - 7. Springer Berlin Heidelberg, pp. 85-108. doi:10.1007/3540-27468-5 7 
Diez, H. V., García, S., Mujika, A., Moreno, A., Oyarzun, D., 2016. Virtual training of fire wardens through immersive 3D environments. Proc. 21st Int. Conf. Web3D Technol. - Web3D '16 43-50. doi:10.1145/2945292.2945296

Dorigo, M., Stutzle, T., Birattari, M., 2010. Ant colony optimization, in: Encyclopedia of Machine Learning. Springer, pp. 36-39.

Eastman, C., Teicholz, P., Sacks, R., Liston, K., 2011. BIM Handbook: A Guide to Building Information Modeling for Owners, Managers, Designers, Engineers and Contractors, Building. doi:10.1002/9780470261309

El-Mekawy, M., Östman, A., Hijazi, I., 2012. A Unified Building Model for 3D Urban GIS. ISPRS Int. J. GeoInformation 1, 120-145. doi:10.3390/ijgi1020120

Evans, D.D., 2003. First responders: Problems and solutions: Tactical information. Technol. Soc. 25, 523-528. doi:10.1016/j.techsoc.2003.09.011

Fallah, N., Apostolopoulos, I., 2013. Indoor human navigation systems: A survey. Interact. with ....

Fischer, C., Gellersen, H., 2010. Location and navigation support for emergency responders: A survey. IEEE Pervasive Comput. 9, 38-47. doi:10.1109/MPRV.2009.91

Gambardella, L., Taillard, É., Agazzi, G., 1999. MACSVRPTW: A Multiple Ant Colony System for Vehicle Routing Problems with Time Windows. New Ideas Optim. 1-17. doi:10.1.1.45.5381

Han, L., Potter, S., Beckett, G., Pringle, G., Welch, S., Koo, S.H., Wickler, G., Usmani, A., Torero, J.L., Tate, A., 2010. FireGrid: An e-infrastructure for next-generation emergency response support. J. Parallel Distrib. Comput. 70, 1128-1141.

Inoue, Y., Sashima, A., Ikeda, T., Kurumatani, K., 2008. Indoor Emergency Evacuation Service on Autonomous Navigation System using Mobile Phone. 2008 Second Int. Symp. Univers. Commun. 79-85. doi:10.1109/ISUC.2008.49

Isikdag, U., Zlatanova, S., Underwood, J., 2013. A BIMOriented Model for supporting indoor navigation requirements. Comput. Environ. Urban Syst. 41, 112-123. doi:10.1016/j.compenvurbsys.2013.05.001

Jones, W.W., Holmberg, D.G., Davis, W.D., Evans, D.D., Bushby, S.T., Reed, K. a, 2005. Workshop to define information needed by emergency responders during building emergencies. US Department of Commerce, Technology Administration, National Institute of Standards and Technology.

Kwan, M.P., Lee, J., 2005. Emergency response after 9/11: The potential of real-time 3D GIS for quick emergency response in micro-spatial environments. Comput. Environ. Urban Syst. 29, 93-113. doi:10.1016/j.compenvurbsys.2003.08.002

Lin, Y.-H., Liu, Y.-S., Gao, G., Han, X.-G., Lai, C.-Y., Gu, M., 2013. The IFC-based path planning for 3D indoor spaces. Adv. Eng. Informatics 27, 189-205. doi:10.1016/j.aei.2012.10.001

Maniezzo, V., Gambardella, L.M., Luigi, F. De, 1991. 5 . Ant Colony Optimization 1-21.

Marco Dorogi, Mauro Birattari, and T.S., 2006. Ant Colony Optimization.
Marino, D., 2006. Air Management: Know Your AirConsumption Rate. Fire Eng. Mag.

Mattsson, B., Juås, B., 1997. The importance of the time factor in fire and rescue service operations in Sweden. Accid. Anal. Prev. 29, 849-857. doi:10.1016/S0001-4575(97)00054-7

NFPA, 2013. National Fire Protection Association [WWW Document]. URL http://www.nfpa.org/

Rakkolainen, I., Vainio, T., 2001. A 3D city info for mobile users. Comput. Graph. 25, 619-625. doi:10.1016/S00978493(01)00090-5

Sabri, N.A.M., Basari, A.S.H., Hussin, B., Samah, K.A.F.A., Wahab, Y., 2016. Ant colony-dijkstra's algorithm for evacuation preparedness in high rise buildings, Lecture Notes in Electrical Engineering. Springer International Publishing, Cham. doi:10.1007/978-3-319-24584-3_46

Safety Health and Survival Section International Association of Fire Chiefs, 2012. Rules of Engagement for Structural Firefighting.

Tang, H., Elalouf, A., Levner, E., Cheng, T.C.E., 2014. Efficient computation of evacuation routes on a threedimensional geometric network. Comput. Ind. Eng. 76, 231242. doi:10.1016/j.cie.2014.08.003

Tashakkori, H., Rajabifard, A., Kalantari, M., 2015a. A new 3D indoor/outdoor spatial model for indoor emergency response facilitation. Build. Environ. 89, 170-182. doi:10.1016/j.buildenv.2015.02.036

Tashakkori, H., Rajabifard, A., Kalantari, M., Aleksandrov, M., 2015b. Indoor Incident Situation Awareness Using 3D Indoor/Outdoor Spatial City Model, in: IEEE Proceedings, The 2nd International Conference on Information and Communication Technologies for Disaster Management. Rennes, France.

Thill, J.C., Dao, T.H.D., Zhou, Y., 2011. Traveling in the threedimensional city: Applications in route planning, accessibility assessment, location analysis and beyond. J. Transp. Geogr. 19, 405-421. doi:10.1016/j.jtrangeo.2010.11.007

Wu, C.-H., Chen, L.-C., 2012. 3D spatial information for firefighting search and rescue route analysis within buildings. Fire Saf. J. 48, 21-29. doi:10.1016/j.firesaf.2011.12.006 